\title{
Intermittent chaos driven by nonlinear Alfvén waves
}

\author{
E. L. Rempel, A. C.-L. Chian, A. J. Preto, and S. Stephany \\ National Institute for Space Research (INPE) and World Institute for Space Environment Research (WISER), P.O. Box 515, \\ São José dos Campos-SP, 12227-010, Brazil
}

Received: 14 September 2004 - Revised: 9 December 2004 - Accepted: 14 December 2004 - Published: 16 December 2004

Part of Special Issue "Advances in space environment turbulence"

\begin{abstract}
We investigate the relevance of chaotic saddles and unstable periodic orbits at the onset of intermittent chaos in the phase dynamics of nonlinear Alfvén waves by using the Kuramoto-Sivashinsky (KS) equation as a model for phase dynamics. We focus on the role of nonattracting chaotic solutions of the KS equation, known as chaotic saddles, in the transition from weak chaos to strong chaos via an interior crisis and show how two of these unstable chaotic saddles can interact to produce the plasma intermittency observed in the strongly chaotic regimes. The dynamical systems approach discussed in this work can lead to a better understanding of the mechanisms responsible for the phenomena of intermittency in space plasmas.
\end{abstract}

\section{Introduction}

Intermittent fluctuations are constantly encountered in space plasmas, as reported in several works based on the analysis of solar wind data both at the ecliptic (Burlaga, 1991; Marsch and Tu, 1993; Bruno et al., 2003) and high heliographic latitude (Ruzmaikin et al., 1995; Pagel and Balogh, 2003). Intermittent events are characterized by time series that display time intervals with low variabilities interrupted by bursts of very high variabilities. As a consequence, the associated probability density functions (PDF's) are non-Gaussian. It has been shown by Vörös et al. (2002) and Dorotovic and Vörös (2004) that intermittent fluctuations in the solar wind affect the geomagnetic response. By comparing solar wind data obtained from the ACE spacecraft with plasma sheet data from the Geotail mission, Dorotovic and Vörös (2004) suggested that the non-Gaussian characteristics of the PDF's of solar wind data can be interconnected to the occurrence of intermittency in the magnetic fluctuations in the plasma sheet. Thus, the study of intermittent phenomena in solar

Correspondence to: E. L. Rempel

(erico@dge.inpe.br) wind plasmas is essential for a better understanding of the solar wind-magnetosphere coupling processes.

The fluctuations of the plasma velocity and magnetic field as revealed by interplanetary data records are frequently well correlated, which is a signature of the presence of Alfvén waves in the solar wind (Belcher and Davis, 1971; Goldstein and Roberts, 1995). Alfvén waves are low-frequency electromagnetic waves in a plasma with a background magnetic field. From a linear analysis of MHD equations, the dispersion relation of the Alfvén wave is found as $\omega=k_{\|} v_{A}$, where $k_{||}$is the component of the wave vector $\boldsymbol{k}$ parallel to $\boldsymbol{B}_{0}$, and $v_{A}=B_{0} /\left(\mu_{0} \rho_{0}\right)^{1 / 2}$ is the Alfvén velocity, where $B_{0}$ is the strength of the ambient magnetic field, $\mu_{0}$ is the permeability of vacuum and $\rho_{0}$ is the average mass density of the plasma. The perturbation of the fluid velocity $\boldsymbol{u}$ relates to the magnetic field's perturbation vector $\boldsymbol{b}=\delta \boldsymbol{B}_{0}$ by $\boldsymbol{u}=\mp \boldsymbol{b} /\left(\mu_{0} \rho_{0}\right)^{1 / 2}$, where the upper (lower) sign refers to the case $\boldsymbol{k} \cdot \boldsymbol{B}_{0}>0$ $\left(\boldsymbol{k} \cdot \boldsymbol{B}_{0}<0\right)$. Thus, $\boldsymbol{u}$ and $\boldsymbol{b}$ are parallel/antiparallel and proportional to each other, and the plasma oscillates with the magnetic field lines.

As shown by Lefebvre and Hada (2000), by assuming weak instability the dynamics of quasiparallel Alfvén waves can be studied by a complex Ginzburg-Landau (CGL) equation. The complex Ginzburg-Landau equation has been one of the most widely studied nonlinear equations in the last decades (see Cross and Hohenberg, 1993; Bohr et al., 1998; Aranson and Kramer, 2002, and references therein). It describes the slow modulation of a periodic pattern in space and time near the threshold of an instability, where a band of modes become unstable.

In this work we study the phase dynamics of nonlinear Alfvén waves modeled by the CGL equation. Phase dynamics is of particular interest in systems modeled by the CGL equation, since for a range of values of the control parameters the dynamics of perturbations of traveling wave solutions is essentially determined by variations of the phase alone, and can exhibit "phase turbulence". In such regimes the amplitudes are essentially constant and the phase dynamics satisfies the Kuramoto-Sivashinsky (KS) equation (Kuramoto 
and Tsuzuki, 1976; Cross and Hohenberg, 1993; Bohr et al., 1998; Aranson and Kramer, 2002; van Baalen, 2004). The study of phase dynamics can elucidate important nonlinear phenomena observed in space plasmas. Finite correlation of phases in MHD waves upstream of Earth's bow shock was found in Geotail magnetic field data, indicating that nonlinear interactions between the waves are in progress (Hada et al., 2003). This bears important implications in discussions of various transport processes of charged particles in space. In He and Chian (2003) imperfect phase synchronization in a nonlinear drift wave system was shown to be responsible for the origin of bursts in wave energy in a turbulent state. Here we focus on the characterization of phase intermittency in the KS equation. We first describe how the fluctuations of the phase can evolve from periodic to chaotic behavior through a sequence of bifurcations as the viscosity is varied. We show that in the KS equation, chaotic attractors coexist with nonattracting chaotic sets responsible for transient chaotic behavior. The collision of a weak chaotic attractor with an unstable periodic orbit leads to the generation of a strong chaotic attractor, in an event known as interior crisis. The post-crisis strong chaotic attractor can be decomposed into two nonattracting chaotic sets, responsible for the generation of intermittent time series.

Section 2 of this paper contains a brief introduction to basic concepts on nonlinear dynamical systems. In Sect. 3 we describe the numerical solutions of the KS equation by the Galerkin spectral method. In Sect. 4 we present our analysis of attracting and nonattracting chaotic sets in the KS equation. The conclusions are given in Sect. 5.

\section{Basic concepts of nonlinear dynamics}

In this section we review some basic concepts of nonlinear dynamical systems (Parker and Chua, 1989; Ott, 1993; Alligood et al., 1996) that are essential for understanding the remaining of this paper.

We consider dissipative dynamical systems described by autonomous systems of ODE's,

$\dot{x}=f(x)$,

where $\boldsymbol{x}$ is an $n$-dimensional vector, $\boldsymbol{f}$ is a vector function and the dot denotes derivative with respect to time. A "flow" $f_{t}\left(\boldsymbol{x}_{\mathbf{0}}\right)$ is the solution of Eq. (1) for an initial condition $\boldsymbol{x}_{\mathbf{0}}$ after certain time $t$. The components of the vector "state variable" $\boldsymbol{x}$ define a "phase space", where the flow of $\boldsymbol{x}_{0}$ is plotted for increasing values of $t$, generating the "orbit" of $x_{0}$.

A "fixed point" of Eq. (1) is a constant solution, i.e. a point $\overline{\boldsymbol{x}}$ for which $\dot{\boldsymbol{x}}=0$, or equivalently, $f_{t}(\overline{\boldsymbol{x}})=\overline{\boldsymbol{x}}$ for all $t$. A "periodic orbit" is a solution of Eq. (1) that always repeats its behavior after a fixed time interval, i.e. $f_{t}(\boldsymbol{x})=f_{t+T}(\boldsymbol{x})$ for all $t$ and some minimum period $T>0$.

Dissipative systems are characterized by the presence of "attractors". An attractor is a subset of the phase space that attracts almost all the initial conditions in a certain neighborhood, that is, the limit set of the orbits of initial conditions in the neighborhood as time tends to $+\infty$ is the attractor (Grebogi et al., 1984). Attractors may be simple sets like a fixed point or periodic orbit, but can also have nonelementary geometrical properties such as noninteger fractal dimension, in which case the attractor is called "strange" (Grebogi et al., 1984, 1987b). Fractal sets display scale invariance, which implies that continuous blow-up of a tiny portion of the set reveal self-similar structures on arbitrarily small scales (Ott, 1993).

Strange attractors are typically (but not always) "chaotic" (Grebogi et al., 1987b). The orbits of random initial conditions on a chaotic attractor will display aperiodic behavior and "sensitive dependence on initial conditions", which means that nearby orbits will diverge exponentially with time. The average rate of divergence can be measured by the "Lyapunov exponent". Let $\Delta_{0}$ be a small distance separating two initial conditions on the chaotic attractor at $t=0$. Then for increasing $t$ the orbits of the two points will diverge on average as $\Delta_{t} \sim \Delta_{0} \exp (\lambda t)$, where $\lambda$ is the Lyapunov exponent (Grebogi et al., 1987b). For systems with $n$-dimensional phase space there are $n$ Lyapunov exponents which measure the rate of divergence/convergence on $n$ orthogonal directions.

Chaotic sets are not necessarily attracting sets. A strange set $\Lambda$ might be chaotic and nonattracting. That means that the orbits of typical initial conditions in the vicinity of $\Lambda$ are eventually repelled from it. Nevertheless, $\Lambda$ contains a chaotic orbit (an aperiodic orbit with at least one positive Lyapunov exponent) (Nusse and Yorke, 1989). If the chaotic orbit has also one negative Lyapunov exponent the nonattracting chaotic set is known as "chaotic saddle". Chaotic saddles, as well as chaotic attractors, contain an infinite number of unstable periodic orbits (UPO's).

In nonlinear dynamical systems, as one varies some control parameter present in the model equations some dynamical changes can occur, such as creation/destruction of fixed points and periodic orbits or loss of stability of attracting sets. Thus, periodic attractors can lose their asymptotic stability and become unstable periodic orbits. Similarly, chaotic attractors can lose their attracting nature and become nonattracting chaotic sets, or chaotic saddles. The qualitative changes in the behavior of solutions of dynamical systems as a control parameter is varied are called "bifurcations". When the changes in the phase portrait involve merely the local vicinity of fixed points or periodic orbits, one has a "local bifurcation". Large changes in the topology of the system are called "global bifurcations". An example is the "interior crisis" discussed in this paper, whereby a chaotic attractor is suddenly enlarged. The bifurcations of a dynamical system can be represented in a "bifurcation diagram", in which the values of one of the state variables are plotted as a function of one control parameter.

A classical technique to analyze nonlinear dynamical systems is the "Poincaré map". It replaces the flow of an $n^{\text {th }}$-order continuous-time system with an $(n-1)^{\text {th }}$-order 
discrete-time system, simplifying the analysis and visualization of the dynamics. There are different forms of defining a Poincaré map. At the end of the next section we exemplify one of them.

\section{The Kuramoto-Sivashinsky equation}

The derivative nonlinear Schrödinger equation (DNLS) (Rogister, 1971; Mjolhus and Wyller, 1988) describes the dynamics of quasiparallel Alfvén waves of moderate amplitudes in a finite- $\beta$ plasma, taking into account weak linear dispersion. To describe patterns formed by Alfvén waves subject to damping and growth rate, Lefebvre and Hada (2000) used a model based on a modified version of the DNLS equation, including linear growth rate, linear dissipation and nonlinear Landau damping. Assuming weak instability, Lefebvre and Hada (2000) proposed a further simplification to an envelope equation by writing the complex transverse magnetic field $B=B_{y}+i B_{z}$ as $B(x, t)=\psi(\xi, t) \exp \left[i\left(k_{c} x-\omega_{c} t\right)\right]$, where $\omega_{c}=\omega\left(k_{c}\right), \xi=x-v\left(k_{c}\right) t$ and $k_{c}$ is the most unstable mode. Assuming $k_{c}>0$ (left-hand polarization) the resulting equation for $\psi$ is given by

$\partial_{t} \psi=\psi+\left(1-i b_{1}\right) \partial_{\xi \xi} \psi-i \psi\left(b_{2}-H\right)|\psi|^{2}$,

where $b_{1}$ measures the strength of the dispersion and $b_{2}$ the effects of nonlinearity. The term $H$ denotes a function that accounts for the nonlinear Landau damping (Mjolhus and Wyller, 1988). Equation (2) falls into the general class of the complex Ginzburg-Landau equation. The envelope $\psi$ is a complex variable. By writing $\psi=R \exp (i \Phi)$ and assuming small perturbations $R=R_{0}+r, \Phi=\Phi_{0}+\phi$, it is possible to derive an equation for the phase $\phi$ of the CGL equation. As shown by Kuramoto and Tsuzuki (1976) and Aranson and Kramer (2002), the phase equation is given by the KuramotoSivashinsky equation. Here we study the following form of the KS equation (LaQuey et al., 1975; Christiansen et al., 1997; Chian et al., 2002; Rempel and Chian, 2003)

$\partial_{t} \phi=-\partial_{x}^{2} \phi-v \partial_{x}^{4} \phi-\partial_{x} \phi^{2}$,

where $v$ is a damping parameter representing viscosity and we assume that $\phi(x, t)$ is subject to periodic boundary conditions

$\phi(x, t)=\phi(x+2 \pi, t)$.

To obtain the numerical solution of Eq. (3) we use the Galerkin method (Gottlieb and Orszag, 1977), by applying a Fourier decomposition for the function $\phi(x, t)$

$\phi(x, t)=\sum_{k=-\infty}^{\infty} b_{k}(t) e^{i k x}$.

After substituting Eq. (5) into Eq. (3) and solving the derivatives one obtains

$\sum_{k=-\infty}^{\infty} \dot{b}_{k} e^{i k x}=\sum_{k=-\infty}^{\infty} k^{2} b_{k} e^{i k x}-v \sum_{k=-\infty}^{\infty} k^{4} b_{k} e^{i k x}$

$$
-\sum_{k=-\infty}^{\infty} \sum_{m=-\infty}^{\infty} i(k+m) b_{k} b_{m} e^{i x(k+m)} .
$$

Next, multiply Eq. (6) by $e^{-i l x}$, with $l=-\infty, \ldots, \infty$, and integrate in the spatial domain,

$$
\begin{gathered}
\int_{0}^{2 \pi} \sum_{k=-\infty}^{\infty} \dot{b}_{k} e^{i x(k-l)} d x=\int_{0}^{2 \pi} \sum_{k=-\infty}^{\infty} k^{2} b_{k} e^{i x(k-l)} d x \\
-\int_{0}^{2 \pi} v \sum_{k=-\infty}^{\infty} k^{4} b_{k} e^{i x(k-l)} d x \\
-\int_{0}^{2 \pi} \sum_{k=-\infty}^{\infty} \sum_{m=-\infty}^{\infty} i(k+m) \\
\times b_{k} b_{m} e^{i x(k+m-l)} d x .
\end{gathered}
$$

Each integral of the exponential functions in Eq. (7) vanishes except when the exponent is zero. The three first exponents are zero when $k=l$. For the nonlinear term the exponent is null when $k=l-m$. After solving the integrals, Eq. (7) becomes

$\begin{aligned} \dot{b}_{k} & =\left(k^{2}-v k^{4}\right) b_{k}-i k \sum_{m=-N}^{N} b_{m} b_{k-m}, \\ k & =-N, \ldots, N,\end{aligned}$

where $N$ is the truncation order. Our choice of $N$ is explained later in this section.

The coefficients $b_{k}$ in Eq. (8) are complex. We can simplify our analysis by restricting to the subspace of odd solutions, $\phi(x, t)=-\phi(-x, t)$. It is possible to prove that if an initial condition $\phi(x, 0)$ is an odd function, the solution of Eq. (3) is odd for all time (Temam, 1988)

$\phi(x, t)=-\phi(-x, t), \quad \forall x, t$.

The Fourier transform of an odd function has purely imaginary coefficients, so we can represent odd functions by assuming that $b_{k}(t)$ are purely imaginary, setting

$b_{k}=-\frac{1}{2} i a_{k}$,

where $a_{k}$ are real numbers. After substituting Eq. (10) into Eq. (8), we obtain

$\dot{a}_{k}=\left(k^{2}-\nu k^{4}\right) a_{k}-\frac{k}{2} \sum_{m=-N}^{N} a_{m} a_{k-m}$,

$k=-N, \ldots, N$.

Equation (11) contains unnecessary operations. Since $\phi(x, t)$ is real, $-i a_{k}=i a_{-k}$, and it is not necessary to compute the modes with negative $k$. Moreover, $a_{k}=0$ for $|k|>N$, and some operations in the nonlinear term can be dropped. Thus Eq. (11) can be written in the form

$$
\begin{aligned}
\dot{a}_{k} & =\left(k^{2}-v k^{4}\right) a_{k}+\frac{k}{2}\left(\sum_{m=k-N}^{-1} a_{-m} a_{k-m}\right. \\
& \left.-\sum_{m=1}^{k-1} a_{m} a_{k-m}+\sum_{m=k+1}^{N} a_{m} a_{m-k}\right),
\end{aligned}
$$




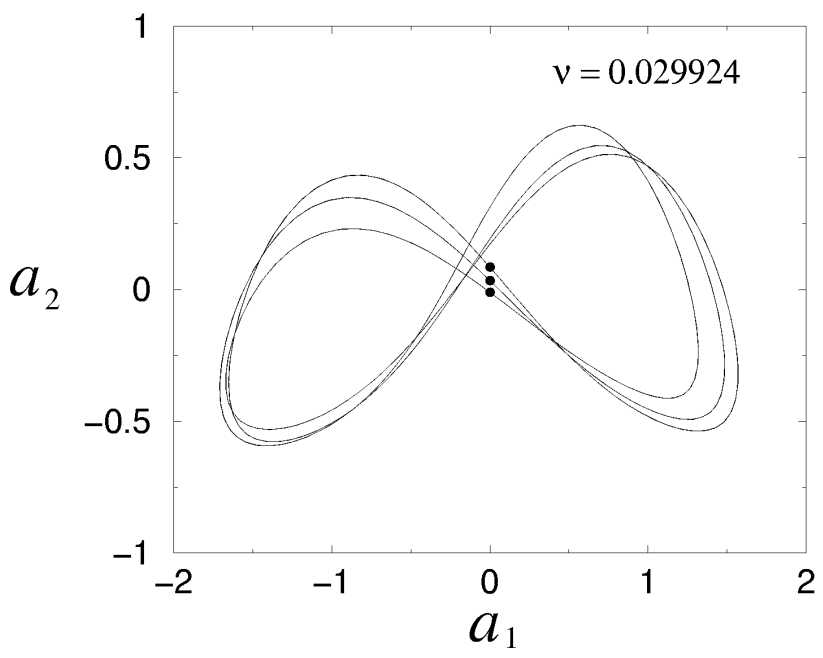

Fig. 1. Period-3 limit cycle solution of Eq. (12) at $\nu=0.029924$ and the corresponding Poincaré points.

with $k=1, \ldots, N$.

The dynamics of the system described by Eq. (12) can be analyzed on a Poincaré section defined by $a_{1}=0$. A trajectory representing the flow of Eq. (12) in a phase space defined by the Fourier modes $a_{k}$, can intersect this Poincaré section in two ways: when $\dot{a}_{1}>0$ (from "left" to "right") or when $\dot{a}_{1}<0$ (from "right" to "left"). We adopt a Poincaré map $P$ defined as the $(N-1)$ dimensional hyperplane given by $a_{1}=0$, with $\dot{a}_{1}>0$, so that a Poincare point is plotted every time the flow of Eq. (12) crosses the Hyperplane $a_{1}=0$ from "left" to "right", as illustrated in Fig. 1 for a period-3 (p-3) limit cycle solution at $\nu=0.029924$.

The choice of the truncation $N$ for the number of modes has obvious implications in the numerical solutions of Eq. (3). High values of $N$ imply high computational cost for the simulations, due to the sums in the nonlinear term in Eq. (12). On the other hand, low values of $N$ may result in a dynamical system whose behavior has no resemblance with the original PDE. A determination of the range of linearly unstable modes is helpful in this case. Considering the linear part of Eq. (12), one finds that the stability eigenvalues are negative for $|k|>1 / \sqrt{v}$ and are positive for $|k|<1 / \sqrt{v}$. Thus, the modes with wave numbers $k$ in the range $[-1 / \sqrt{v}, 1 / \sqrt{v}]$ are linearly unstable. These modes excite the short wavelength (high- $k$ ) modes through the nonlinear term in Eq. (12), and the excitations are dissipated by the high- $k$ modes (Christiansen et al., 1997). Modes with $|k| \gg 1 / \sqrt{v}$ are strongly damped and their amplitudes remain very close to zero for all times, but intermediate modes must be kept in the simulations. Figure 2a depicts the temporal variation of the Fourier modes $a_{k}(t)$ in Eq. (12) for a chaotic regime at $\nu=0.029919$ and $N=16$ modes. Clearly, the energy is concentrated in the low- $k$ long wavelength modes. The corresponding spatiotemporal pattern $\phi(x, t)$ in real space, obtained with Eq. (5), is shown in Fig. 2b. The system dynamics is chaotic in time but coherent in space.
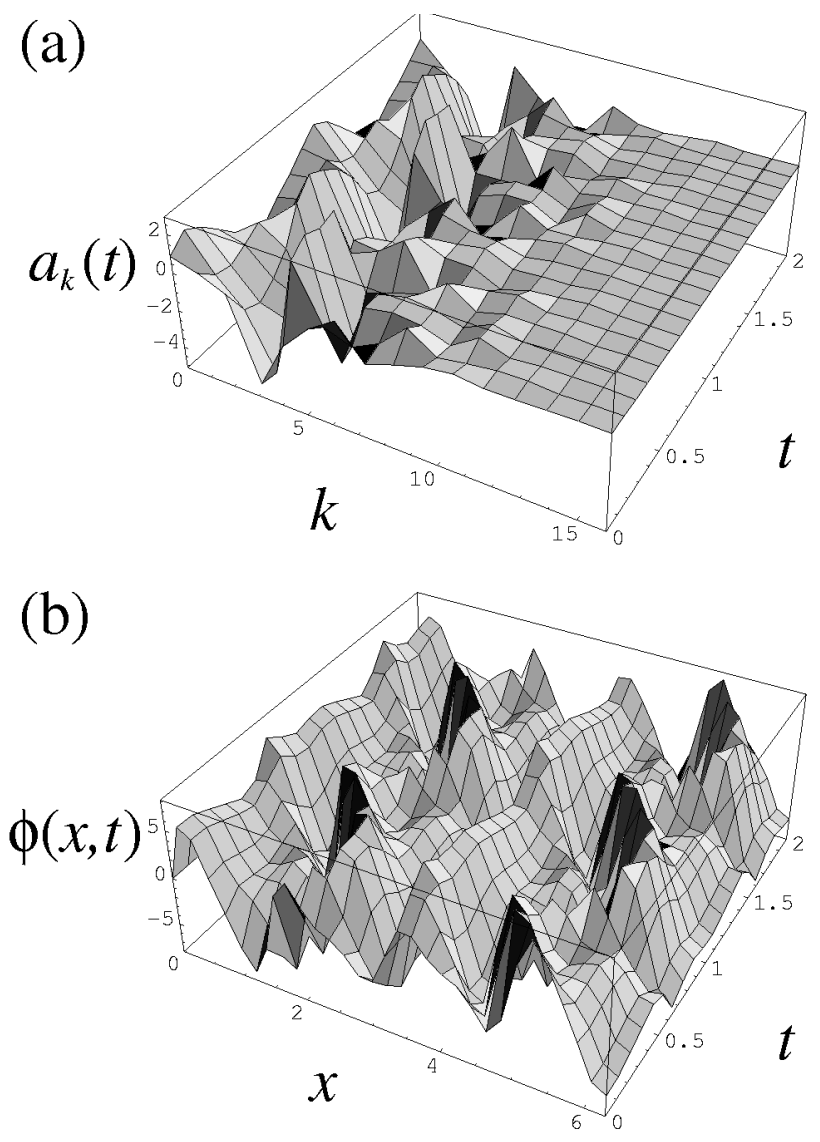

Fig. 2. (a) Temporal variation of the Fourier modes $a_{k}(t)$ in Eq. (12) for $\nu=0.029919$ and $N=16$ modes; (b) the corresponding spatiotemporal pattern of $\phi(x, t)$. The system dynamics is chaotic in time but coherent in space.

\section{Nonlinear dynamics analysis}

\subsection{The bifurcation diagram}

Figure 3a depicts the bifurcation diagram $a_{6}(v)$ for Eq. (12) with $N=16$. The diagram is similar for any choice of $a_{k}$. For each value of $v$ we drop the initial 100 iterations of the Poincaré map before we start plotting. These initial iterations contain the transient dynamics, before the fluctuations of the wave phase converge to a regime where the Poincaré points stay in an attracting subset of the phase space. For this range of $v$, the attracting set can be either chaotic or periodic. The gray area in Fig. 3a depicts another important subset of the phase space representing a chaotic saddle. The trajectories of random initial conditions are first attracted to the vicinity of the chaotic saddle, where they display chaotic behavior for a finite time ("chaotic transient"), before they converge to the attractor (periodic or chaotic). The chaotic saddles are obtained by the PIM triple algorithm (Nusse and Yorke, 1989).

A saddle-node bifurcation at $\nu=v_{\mathrm{SNB}} \approx 0.02992498$, indicated as SNB in Fig. 1, marks the beginning of a "periodic window" in the bifurcation diagram. For $v>v_{\mathrm{SNB}}$, ran- 

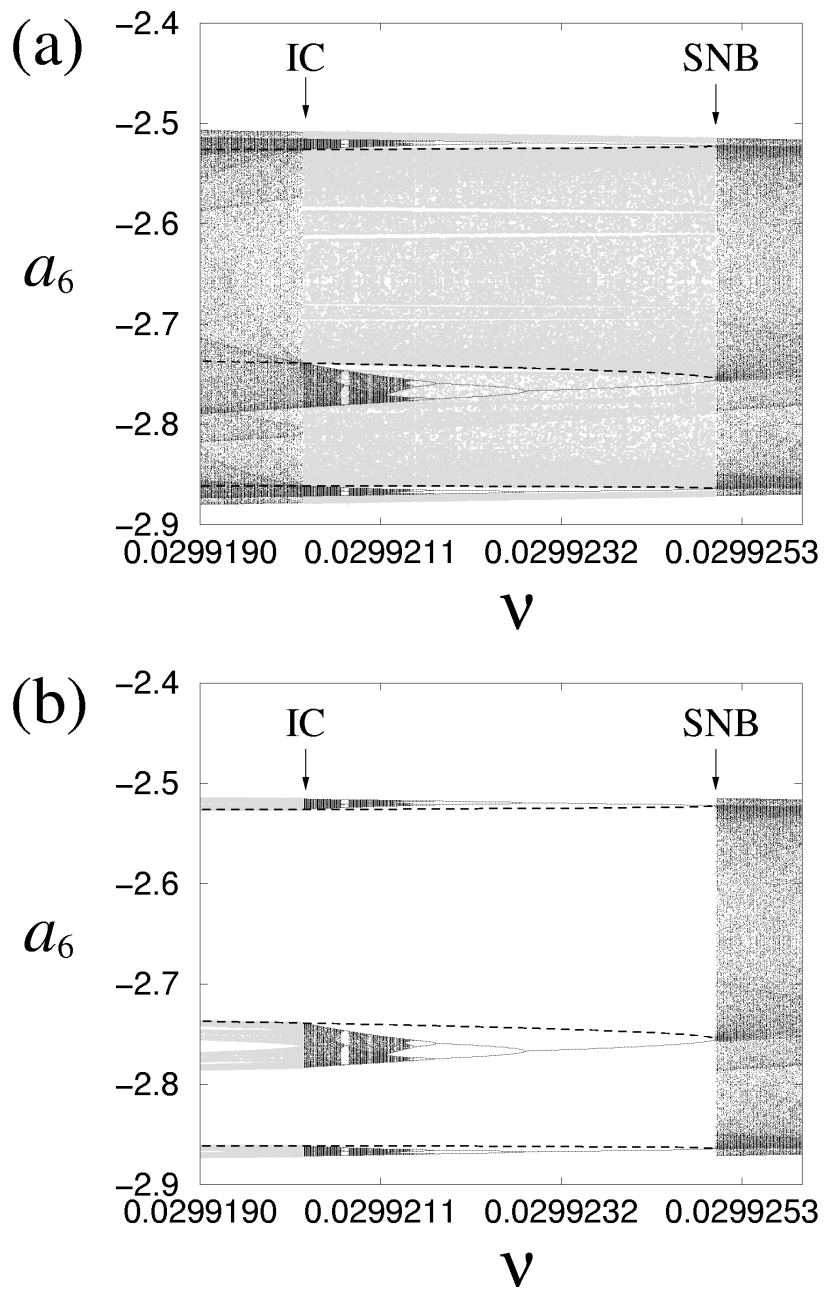

Fig. 3. (a) Variation of $a_{6}$ for the chaotic saddle (gray) as a function of $v$, superimposed by the bifurcation diagram of the attractor (black) in a p-3 periodic window. IC denotes interior crisis and SNB denotes saddle-node bifurcation. The dashed lines denote the p-3 mediating unstable periodic orbit. (b) Same as (a), but depicting the conversion of the three-band weak chaotic attractor into a band chaotic saddle after (to the left of) IC.

dom initial conditions converge to a chaotic attractor, and for $v<v_{\text {SNB }}$ the chaotic attractor no longer exists. At the saddlenode bifurcation the simultaneous creation of a p-3 attractor and a p-3 unstable periodic orbit occurs. The p-3 UPO, found with the Newton method, is represented in Fig. 3a by dashed lines. As the value of $v$ is decreased, the $\mathrm{p}-3$ attractor undergoes a cascade of period-doubling bifurcations, whereby the period of the attractor is successively doubled. As the period tends towards infinity, a chaotic attractor is formed, localized in three separate bands in the bifurcation diagram. We call the region occupied by this "banded" attractor the "band region" (B), and the region occupied by the surrounding chaotic saddle (SCS) the "surrounding region" (S), following reference (Szabó et al., 2000). At $\nu=v_{\mathrm{IC}} \approx 0.02992021$ the chaotic attractor collides with the p-3 UPO created at $\mathrm{SNB}$, called the "mediating unstable periodic orbit" (MPO).
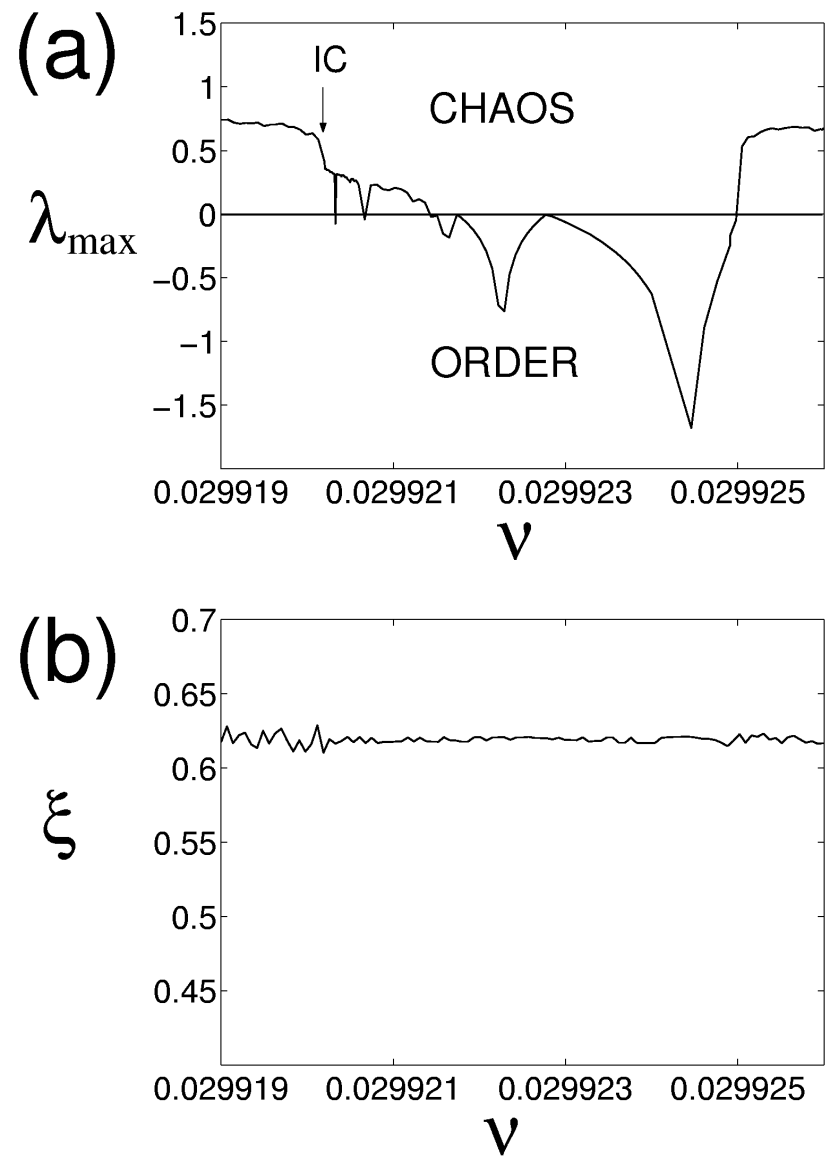

Fig. 4. (a) Variation of the maximum Lyapunov exponent $\lambda$ max with $v$; (b) variation of the correlation length $\xi$ with $v$.

This collision is responsible for an "interior crisis", which is a sudden enlargement in the size of a chaotic attractor (Grebogi et al., 1983). After crisis $\left(v<\nu_{\text {IC }}\right)$, we find a new chaotic saddle embedded in the enlarged chaotic attractor, in the region previously occupied by the "pre-IC" banded chaotic attractor. In contrast with the surrounding chaotic saddle, we call this new chaotic saddle the "band chaotic saddle" (BCS). Figure $3 b$ illustrates the structure of BCS after IC, where the bifurcation diagram for the attractor (black) of Fig. 3a is plotted to the right of the IC point and the band chaotic saddle (gray) is plotted to the left of IC. It is important to stress that although in Fig. 3a the surrounding chaotic saddle is plotted only between points SNB and IC, it is actually present in the entire bifurcation diagram. For $v<v_{\mathrm{IC}}$ and $v>v_{\mathrm{SNB}}$ SCS is a subset of the chaotic attractor.

In Fig. 4a we plot the variation of the maximum Lyapunov exponent $\lambda_{\max }$ of the attracting set as a function of $\nu$. Positive values of $\lambda_{\max }$ indicate the presence of a chaotic attractor, and negative values indicate that the attractor is periodic. Note that $\lambda_{\max }$ jumps abruptly at $\nu_{\mathrm{IC}}$, indicating a sudden increase in the attractor's chaoticity. For that reason, the pre-IC chaotic attractor is called "weak chaotic attractor" and the post-IC attractor, the "strong chaotic attractor". As mentioned before, for the chosen value of the damping 


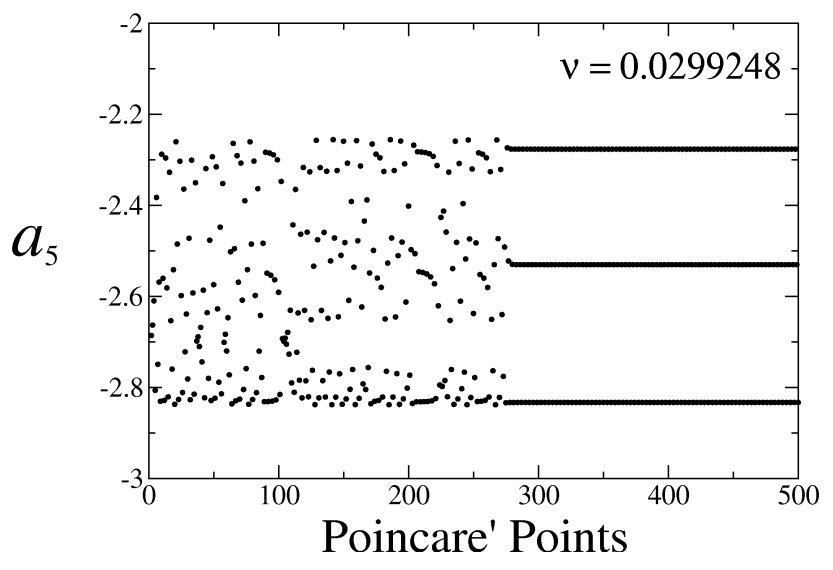

Fig. 5. Transient chaos at $v=0.0299248$.

parameter $v$ and the spatial system size $L=2 \pi$, the dynamics of the Kuramoto-Sivashinsky equation is chaotic in time, but coherent in space. In fact, the spatial coherence remains basically unaltered throughout the whole range of $v$ used in Figs. 3 and 4, as indicated by the correlation length $\xi$ (Morris et al., 1993) in Fig. 4b.

\subsection{Chaotic saddles}

Associated to a chaotic saddle, there are stable and unstable manifolds. The stable manifold is the set of points that converge to the chaotic saddle in forward time dynamics; the unstable manifold is the set of points that converge to the chaotic saddle in the time reversed dynamics. The chaotic saddle lies on the intersection of its stable and unstable manifolds (Nusse and Yorke, 1989; Ziemniak et al., 1994). Inside the periodic window (between SNB and IC in Fig. 3) the trajectories of all initial conditions will eventually converge to an attractor, except for initial conditions lying on the stable manifold of SCS, which is a set of measure zero. Initial conditions close to the stable manifold are first attracted to SCS and stay close to its neighborhood for some time, before they are repelled, following its unstable manifold. The closer an initial condition is to the stable manifold of SCS, the longer its transient time before converging to the attractor (Hsu et al., 1988). In Fig. 5 an example of transient chaos is shown for a time series of Poincaré points at $\nu=0.0299248$, where the phase dynamics converges to a $\mathrm{p}$ 3 attractor. The average transient time $\tau$ can be estimated by taking $N_{0}$ random initial conditions and computing $N_{t}$, the number of trajectories that are still in the transient stage after $t$ iterates. Figure 6 shows a graph of $\log N_{t}$ versus $t$ at $\nu=v_{\text {IC }}$, where $N_{0}=10000$ different initial conditions were used. The graph can be fitted with a straight line of slope $\gamma=-3.59 \times 10^{-2} \pm 1.45 \times 10^{-4}$, which gives an average exit time $\tau=-1 / \gamma \approx 27.9$ (Rempel et al., 2004).

Figure 7 shows a three-dimensional projection $\left(a_{1}, a_{10}\right.$, $a_{16}$ ) of a chaotic saddle defined in the 15-dimensional Poincaré hyperplane at $\nu=0.029925>v_{\mathrm{SNB}}$, to the right of the saddle-node bifurcation in Fig. 3a. This chaotic saddle

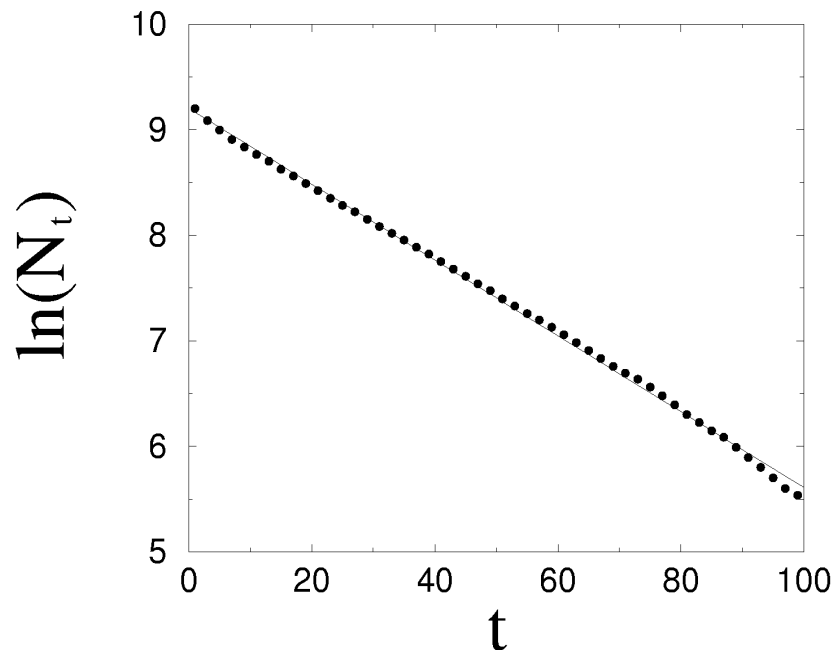

Fig. 6. Exponential decay of $N_{t}$, the number of trajectories inside the restraining region at time $t$, as a function of $t$. The inverse of the slope of the fitted line gives the average exit time $\tau \approx 27.9$.

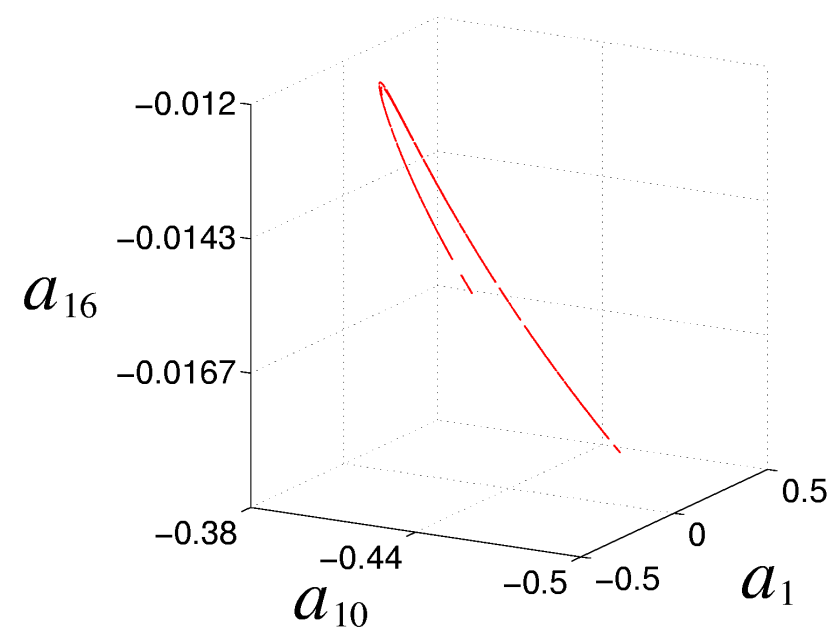

Fig. 7. Three-dimensional projection $\left(a_{1}, a_{10}, a_{16}\right)$ of the chaotic saddle defined in the 15-dimensional Poincaré hyperplane just before the saddle-node bifurcation, at $\nu=0.029925$.

is a continuation of the surrounding chaotic saddle shown in Fig. 3a. The chaotic saddle is not a continuous line. It has many gaps, most of which are not visible in Fig. 7 due to their small size.

\subsection{Interior crisis}

In this section the dynamics near the interior crisis point $v_{\text {IC }}$ is investigated in terms of the role of chaotic saddles and UPOs. For details on the numerical algorithms employed see Rempel and Chian (2003) and Rempel et al. (2004).

At crisis the collision of the mediating UPO with the tree-band weak chaotic attractor at $v_{\text {IC }}$ results in the formation of a single-band strong chaotic attractor. Figure 8 shows a three-dimensional projection $\left(a_{1}, a_{10}, a_{16}\right)$ of the 


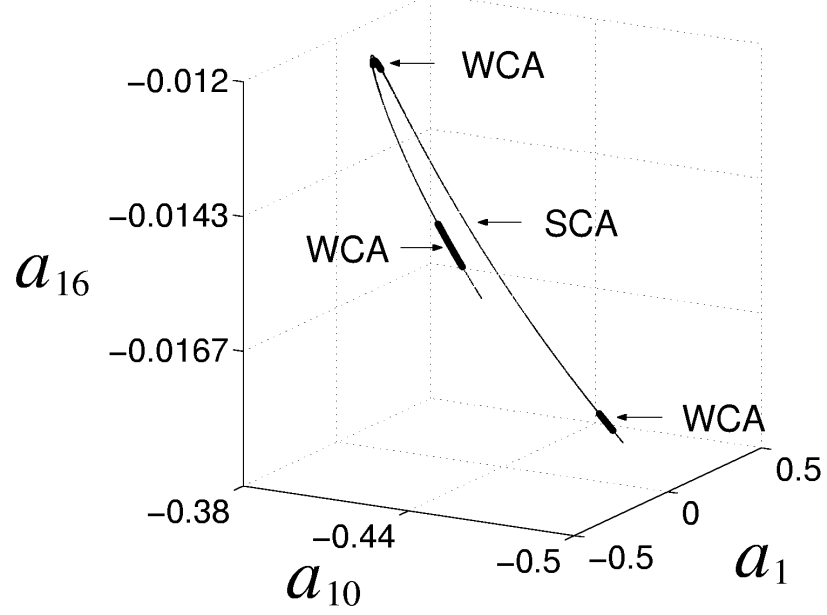

Fig. 8. Three-dimensional projection $\left(a_{1}, a_{10}, a_{16}\right)$ of the strong chaotic attractor (SCA, light line) defined in the 15-dimensional Poincaré hyperplane after crisis at $\nu=0.02992006$, superimposed by the three-band weak chaotic attractor (WCA, dark lines) at crisis $(\nu=0.02992021)$.

Poincaré points of the strong chaotic attractor (SCA, light line) after crisis $(\nu=0.02992006)$, superimposed by the 3band weak chaotic attractor (WCA, dark lines) at crisis $(\nu=0.02992021)$. An estimation of the fractal dimension of the post-crisis chaotic attractor at $v=0.02992006$ using the Kaplan-Yorke formula (Ott, 1993) results in the dimension $D \approx 2.08$. Note the similarity of the strong chaotic attractor with the surrounding chaotic saddle shown in Fig. 7.

In Fig. 9 we plot a two-dimensional projection $\left(a_{5}, a_{6}\right)$ of the upper branch of the chaotic attractor CA (black line) with the surrounding chaotic saddle SCS (red lines) and its stable manifold (blue dots) for (a) $\nu=0.0299211$ (before crisis) and (b) $v=0.02992021$ (at crisis). The upper Poincaré point of the p-3 mediating orbit is represented by the cross. In the Poincaré map, an unstable periodic orbit turns into a set of saddle points with their associated stable and unstable manifolds. The dashed lines in Fig. 9 denote the local branches of the stable manifold (SM) of the mediating orbit (MPO). Note that SCS has a large gap between the two dashed lines, and many other smaller gaps, or discontinuities. Its gaps are due to the horizontal white spaces in the background, which reflect the fractal structure of the stable manifold of SCS. Figure $1 \mathrm{~b}$ reveals that at crisis the $\mathrm{S}$ chaotic saddle and the weak chaotic attractor collide. The collision takes place at the mediating saddle, which belongs to SCS. Likewise, the weak chaotic attractor collides with the stable manifolds of both SCS and MPO.

\subsection{Crisis-induced intermittency}

After the crisis, it is still possible to determine the B and S regions, which are separated by the stable manifold of MPO. Figure 10 shows the chaotic sets for $v=0.02992006$, after the crisis, around the upper branch of the strong chaotic attrac-
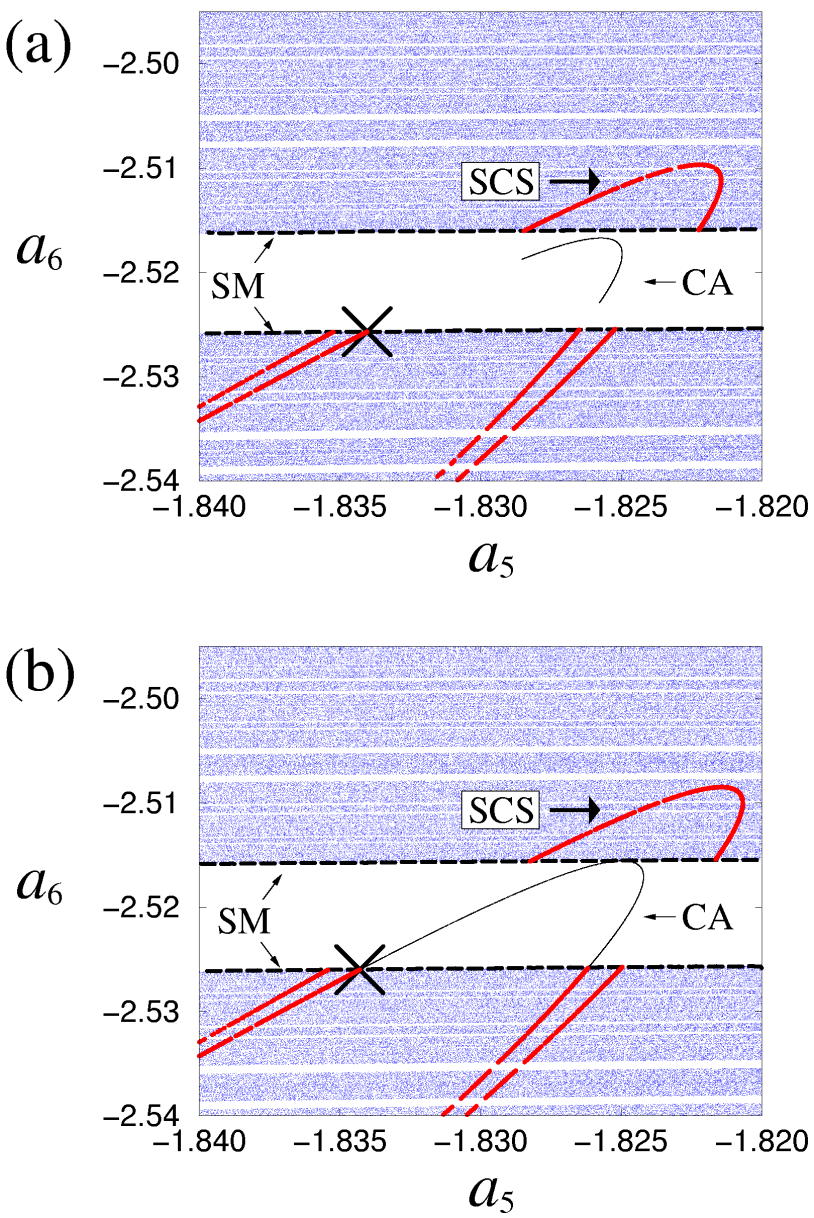

Fig. 9. Plots of the upper branch of the chaotic attractor (CA, black line), the surrounding chaotic saddle (SCS, red lines) and its stable manifolds (blue dots): (a) before the interior crisis, at $\nu=0.0299211$; and (b) at the interior crisis, $v=0.02992021$. The cross denotes one of the Poincaré points of the p- 3 mediating unstable periodic orbit. The dashed lines represent segments of the boundary between the band region and the surrounding region, given by the stable manifolds (SM) of the mediating unstable periodic orbit.

tor shown in Fig. 8. Figure 10a shows the chaotic attractor (CA) and Fig. 10b shows the corresponding B (green lines) and $\mathrm{S}$ (red lines) chaotic saddles. BCS is localized in a region of the phase space previously occupied by the pre-crisis weak chaotic attractor. SCS is the continuation of the precrisis surrounding chaotic saddle. It can be seen from Fig. 10 that the post-crisis $\mathrm{B}$ and $\mathrm{S}$ chaotic saddles are subsets of the strong chaotic attractor. The gaps in SCS and BCS are filled by a set of coupling UPO's created at the crisis (Robert et al., 2000).

The two post-IC chaotic saddles are not attracting, but they exert influence on the dynamics of nearby orbits, since they are responsible for chaotic transients. For $v>v_{\text {IC }}$, trajectories on the chaotic attractor never abandon the band region. For $v$ slightly less than $v_{\text {IC }}$ a trajectory started in the $\mathrm{B}$ region can stay in B for a long time, after which it crosses SM and 

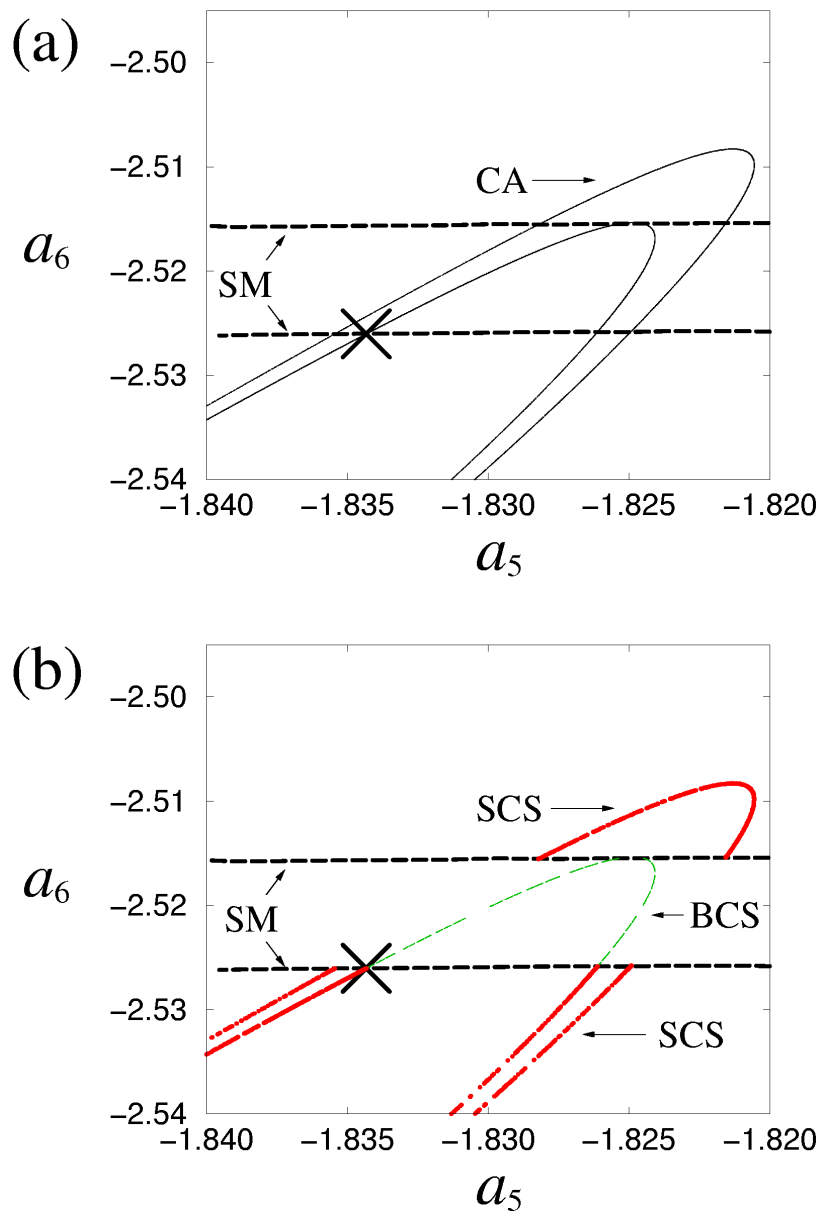

Fig. 10. (a) Upper branch of the chaotic attractor (CA) after the interior crisis, at $\nu=0.02992006$. The dashed lines indicate segments of the stable manifolds (SM) of the mediating unstable periodic orbit (cross); (b) the band chaotic saddle (BCS, green lines) and the surrounding chaotic saddle (SCS, red lines) that compose the chaotic attractor shown in (a).

escapes to region S. Once it is in the surrounding region, the trajectory is in the neighborhood of SCS. Since SCS is nonattracting, after some time the trajectory is "re-injected" into region B. The "jumps" between regions B and S repeat intermittently. This crisis-induced intermittency can be viewed as an alternation between two transient behaviors, in which the trajectory spends a finite time in the vicinity of either $\mathrm{BCS}$ or SCS. These transitions between regions B and S are due to the coupling UPO's that are located within the gaps of BCS and SCS, and establish the dynamical connection between the two chaotic saddles (Szabó et al., 2000; Rempel and Chian, 2004). The crisis-induced intermittency is characterized by time series containing weakly chaotic laminar phases that are randomly interrupted by strongly chaotic bursts, as shown in Fig. 11 for $v=0.02992$. The weakly chaotic laminar phases correspond to the time spent in region $\mathrm{B}$, and the strongly chaotic bursts correspond to the time spent in region $S$.

The average duration of the "laminar" phases in the inter-

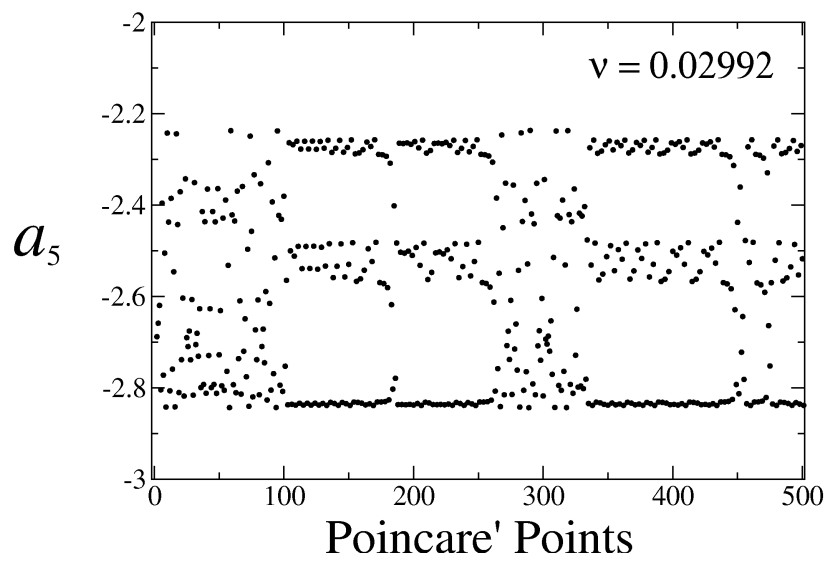

Fig. 11. Crisis-induced intermittency at $v=0.02992$.

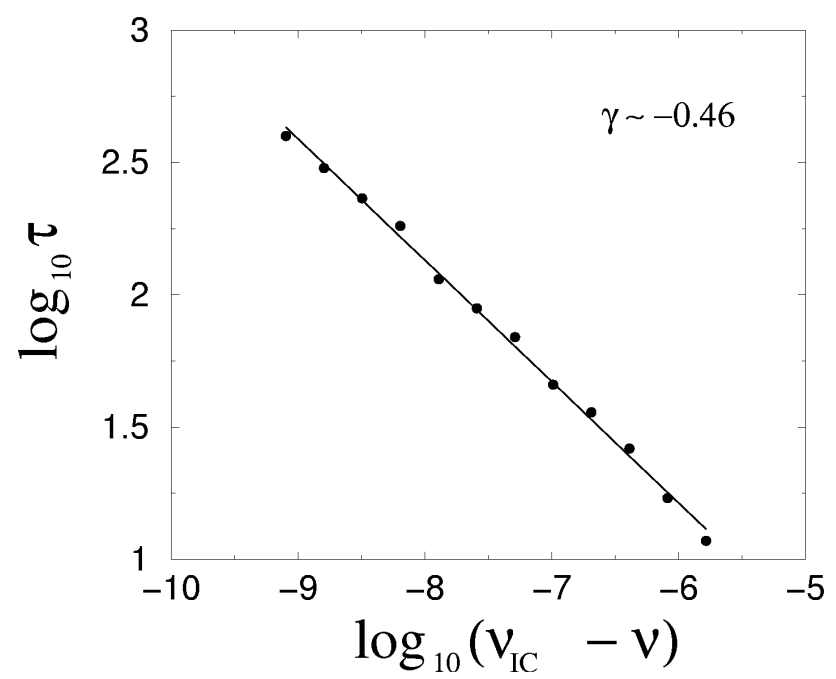

Fig. 12. $\log _{10} \tau$ vs. $\log _{10}\left(v_{\mathrm{IC}}-v\right)$. The solid line with slope $\gamma \approx-0.46$ is a linear fit of the values of the characteristic intermittency time $\tau$ computed from time series (circles).

mittent time series (the "characteristic intermittency time") depends on the value of $v$. Close to $v_{\text {IC }}$ the average time spent in the vicinity of BCS is very long, and decreases as $v$ is decreased away from $\nu_{\text {IC }}$. The characteristic intermittency time (denoted by $\tau$ ) can be obtained as the average over a long time series of the time between switches among regions B and S. Figure 12 is a plot of $\log _{10} \tau$ vs. $\log _{10}\left(v_{\mathrm{IC}}-v\right)$, where the solid line with slope $\gamma \approx-0.46$ is a linear fit of the values of the characteristic intermittency time computed from time series (circles). Figure 12 reveals that the characteristic time $\tau$ decreases with the distance from the critical parameter value $\nu_{\mathrm{IC}}$ following a power-law decay, $\tau \sim\left(\nu_{\mathrm{IC}}-v\right)^{\gamma}$, as expected (Grebogi et al., 1987a). 


\section{Conclusions}

We have investigated the relevance of chaotic saddles and unstable periodic orbits at the onset of intermittent chaos in the phase dynamics of nonlinear Alfvén waves by using the Kuramoto-Sivashinsky equation as a model equation. We described how a strong chaotic attractor formed after an interior crisis can be naturally decomposed into two nonattracting chaotic sets, known as chaotic saddles, dynamically linked by a set of coupling unstable periodic orbits. The perturbed wave phase oscillates irregularly, switching intermittently from the neighborhood of one nonattracting chaotic set to the other.

The dynamical scenario described in this paper reflects a situation of intermittency in chaotic spatiotemporal systems that is still distant from the well-developed turbulent regimes found in space plasmas, where one expects a huge number of modes to become actively involved in the dynamics. Nevertheless, we believe the dynamical systems approach discussed in this work may be helpful to understand more complex cases. Several authors have searched for evidence of deterministic chaos in space plasmas. Pavlos et al. (1992) found evidence of a low-dimensional chaotic attractor in the solar wind through the determination of the correlation dimension from time series of the magnetic field obtained by the IMP-8 spacecraft at $1 \mathrm{AU}$. A low value for the correlation dimension obtained from solar wind data was also reported in other works (Marsch and Tu, 1993; Burlaga, 1995). Macek and Obojska (1997), Macek and Redaelli (2000) and Redaelli and Macek (2001) estimated the Lyapunov exponents, fractal dimension and the Kolmogorov entropy from solar wind data obtained by the Helios 1 spacecraft in the inner heliosphere, concluding that the solar wind is likely a deterministic chaotic system. Sorriso-Valvo et al. (2001) studied plasma intermittency by using a simplified shell model which mimics 3-D MHD equations. They showed that, just like in solar wind data, the intermittency displayed by the shell model is characterized by a strong departure from Gaussian behavior in the probability density functions for the velocity and magnetic field fluctuations at small scales. Although it is not possible to state that the erratic fluctuations observed in the real world are simple manifestations of chaos, in the works mentioned above the behavior observed in nature seems to have a strong deterministic component. Thus, the study of dynamical systems can lead to a better understanding of the mechanisms responsible for the onset of intermittent turbulence in space plasmas. In fact, space data is a mixture of both stationary and nonstationary components. Therefore we can perform space data analysis using either deterministic or statistical techniques. Most works on solar wind intermittency are based on statistical analysis (Burlaga, 1991; Marsch and Tu, 1993; Vörös et al., 2002; Bruno et al., 2003; Dorotovic and Vörös, 2004). Our theoretical study of solar wind intermittency based on chaos theory provides new insights to extend the deterministic analysis of solar wind data (Macek and Obojska, 1997; Macek and Redaelli, 2000; Redaelli and Macek, 2001) to deepen our understanding of solar wind in- termittency. In particular, our theoretical results can be used to reconstruct chaotic transients in phase space from space data and to calculate the average duration of laminar phases in solar wind intermittency.

Acknowledgements. This work is supported by FAPESP and CNPq.

Edited by: T. Passot

Reviewed by: two referees

\section{References}

Alligood, K. T., Sauer, T. D., and Yorke, J. A.: Chaos: an introduction to dynamical systems, Springer Verlag, New York, 1996.

Aranson, I. S. and Kramer, L.: The world of the complex GinzburgLandau equation, Rev. Mod. Phys., 74, 99-143, 2002.

Belcher, J. W. and Davis, L.: Large-amplitude Alfvén waves in interplanetary medium, 2, J. Geophys. Res., 76, 3534-3563, 1971.

Bohr, T., Jensen, M. H., Paladin, G., and Vulpiani, A.: Dynamical systems approach to turbulence, Cambridge University Press, Cambridge, 1998.

Bruno, R., Carbone, V., Sorriso-Valvo, L., and Bavassano, B.: Radial evolution of solar wind intermittency in the inner heliosphere, J. Geophys. Res., 108, 1130-1145, 2003.

Burlaga, L. F.: Intermittent turbulence in the solar wind, J. Geophys. Res., 96, 5847-5851, 1991.

Burlaga, L. F.: Interplanetary Magnetohydrodynamics, Oxford University Press, Oxford, 1995.

Chian, A. C.-L., Rempel, E. L., Macau, E. E., Rosa, R. R., and Christiansen, F.: High-dimensional interior crisis in the Kuramoto-Sivashinsky equation, Phys. Rev. E, 65, 035 203(R), 2002.

Christiansen, F., Cvitanović, P., and Putkaradze, V.: Spatiotemporal chaos in terms of unstable recurrent patters, Nonlinearity, 10, 5570, 1997.

Cross, M. C. and Hohenberg, P. C.: Pattern formation outside of equilibrium, Rev. Mod. Phys., 65, 851-1112, 1993.

Dorotovic, I. and Vörös, Z.: Examining the role of turbulence in the solar wind-magnetosphere interaction processes, Proceedings IAU Symposium, 223, in press, 2004.

Goldstein, M. L. and Roberts, D. A.: Magnetohydrodynamic turbulence in the solar wind, Ann. Rev. Astron. Astrophys., 33, 283 325, 1995 .

Gottlieb, D. and Orszag, S. A.: Numerical analysis of spectral methods: theory and applications, Society for Industrial and Applied Mathematics, Philadelphia, 1977.

Grebogi, C., Ott, E., and Yorke, J. A.: Crises, sudden changes in chaotic attractors, and transient chaos, Physica D, 7, 181-200, 1983.

Grebogi, C., Ott, E., Pelikan, S., and Yorke, J. A.: Strange attractors that are not chaotic, Physica D, 13, 261-268, 1984.

Grebogi, C., Ott, E., Romeiras, F., and Yorke, J. A.: Critical exponents for crisis-induced intermittency, Phys. Rev. A, 36, 53655380, 1987a.

Grebogi, C., Ott, E., and Yorke, J. A.: Chaos, strange attractors, and fractal basin boundaries in nonlinear dynamics, Science, 238, 585-718, 1987b.

Hada, T., Koga, D., and Yamamoto, E.: Phase coherence of MHD waves in the solar wind, Spa. Sci. Rev., 107, 463-466, 2003. 
He, K. F. and Chian, A. C.-L.: On-off collective imperfect phase synchronization and bursts in wave energy in a turbulent state, Phys. Rev. Lett., 3, 034 102, 2003.

Hsu, G.-H., Ott, E., and Grebogi, C.: Strange saddles and the dimensions of their invariant manifolds, Phys. Lett. A, 127, 199204, 1988.

Kuramoto, Y. and Tsuzuki, T.: Persistent propagation of concentration waves in dissipative media far from thermal equilibrium, Prog. Theor. Phys., 55, 356-369, 1976.

LaQuey, R. E., Mahajan, S. M., Rutherford, P. H., and Tang, W. M.: Nonlinear saturation of the trapped-ion-mode, Phys. Rev. Lett., 34, 391-394, 1975.

Lefebvre, B. and Hada, T.: Spatio-temporal behavior of a driven system of MHD waves, Eos Transactions of AGU, Fall Meeting Supplement, 81, Abstract SM62A-09, 2000.

Macek, W. M. and Obojska, L.: Fractal analysis of the solar wind flow in the inner heliosphere, Chaos Solitons Fractals, 8, 16011607, 1997.

Macek, W. M. and Redaelli, S.: Estimation of the entropy of the solar wind flow, Phys. Rev. E, 62, 6496-6504, 2000.

Marsch, E. and Tu, C.-Y.: Structure functions and intermittency of velocity fluctuations in the inner solar wind, Ann. Geophysicae, 11, 227-238, 1993.

Mjolhus, E. and Wyller, J.: Nonlinear Alfvén waves in a finite-Beta plasma, J. Plasma Phys., 40, 299-318, 1988.

Morris, S. W., Bodenschatz, E., Cannell, D. S., and Ahlers, G.: Spiral defect chaos in large aspect ratio Rayleigh-Bénard convection, Phys. Rev. Lett., 71, 2026-2029, 1993.

Nusse, H. E. and Yorke, J. A.: A procedure for finding numerical trajectories on chaotic saddles, Physica D, 36, 137-156, 1989.

Ott, E.: Chaos in dynamical systems, Cambridge Univ. Press, Cambridge, 1993.

Pagel, C. and Balogh, A.: Radial dependence of intermittency in the fast polar solar wind magnetic field using Ulysses, J. Geophys. Res., 108, 1012-1028, 2003.

Parker, T. S. and Chua, L. O.: Practical numerical algorithms for chaotic systems, Springer-Verlag, New York, 1989.
Pavlos, G. P., Kyriakou, G. A., Rigas, A. G., Liatsis, P. I., Trochoutsos, P. C., and Tsonis, A. A.: Evidence for strange attractor structures in space plasmas, Ann. Geophysicae, 10, 309-322, 1992.

Redaelli, S. and Macek, W. M.: Lyapunov exponent and entropy of the solar wind flow, Planet. Space Sci., 49, 1211-1218, 2001.

Rempel, E. L. and Chian, A. C.-L.: High-dimensional chaotic saddles in the Kuramoto-Sivashinsky equation, Phys. Lett. A, 319, 104-109, 2003.

Rempel, E. L. and Chian, A. C.-L.: Alfvén chaotic saddles, Int. J. Bifurcation Chaos, 14, in press, 2004.

Rempel, E. L., Chian, A. C.-L., Macau, E. E. N., and Rosa, R. R.: Analysis of chaotic saddles in high-dimensional dynamical systems: the Kuramoto-Sivashinsky equation, CHAOS, 14, 545556, 2004.

Robert, C., Alligood, K. T., Ott, E., and Yorke, J. A.: Explosions of chaotic sets, Physica D, 144, 44-61, 2000.

Rogister, A.: Parallel propagation of nonlinear low-frequency waves in high-Beta plasma, Phys. Fluids., 14, 2733, 1971.

Ruzmaikin, A. A., Feynman, J., Goldstein, B. E., Smith, E. J., and Balogh, A.: Intermittent turbulence in solar wind from the south polar hole, J. Geophys. Res., 100, 3395-3404, 1995.

Sorriso-Valvo, L., Carboni, V., Giulinani, P., Veltri, P., Bruno, R., Antoni, V., and Martines, E.: Intermittency in plasma turbulence, Planet. Space Sci., 49, 1193-1200, 2001.

Szabó, K. G., Lai, Y.-C., Tél, T., and Grebogi, C.: Topological gap filling at crisis, Phys. Rev. E, 61, 5019-5032, 2000.

Temam, R.: Infinite-dimensional dynamical systems in mechanics and physics, Springer-Verlag, New York, 1988.

van Baalen, G.: Phase turbulence in the complex Ginzburg-Landau equation via Kuramoto-Sivashinsky phase dynamics, Commun. Math. Phys., 247, 613-654, 2004.

Vörös, Z., Jankovicová, D., and Kovács, P.: Scaling and singularity characteristics of solar wind and magnetospheric fluctuations, Nonl. Proc. Geophys., 9, 149-162, 2002.

Ziemniak, E. M., Jung, C., and Tél, T.: Tracer dynamics in open hydrodynamical flows as chaotic scattering, Physica D, 76, 123 146, 1994. 